\title{
Hip Hop Chicana en Los Ángeles: saberes y luchas del barrio latino en femenino ${ }^{2}$
}

\author{
Chicana Hip Hop in Los Angeles: Knowledges and Struggles of the Latin Neighborhood in the Feminine \\ Hip Hop Chicana em Los Ángeles: saberes e lutas do bairro latino em feminino
}

\begin{abstract}
Resumen
El presente artículo de investigación realiza un acercamiento al mundo del hip hop chicana en la ciudad de Los Ángeles para ilustrar las motivaciones, estrategias y luchas de las mujeres que encuentran su latir particularmente en el rap. En primer lugar, se presenta una discusión sobre el hip hop chicano en la ciudad de Los Ángeles, su relevancia para la juventud del barrio postindustrial y la importancia de la producción femenina como elemento faltante para una representación más integral de esa experiencia. En segundo lugar, se profundiza en los conflictos que estas mujeres experimentan y las estrategias que desarrollan dentro de este movimiento cultural. Finalmente, se plantea la discusión del hip hop chicana como activismo. Los argumentos aquí presentados vienen acompañados de los testimonios recolectados en las entrevistas hechas a cinco raperas chicanas y algunos fragmentos de las letras de las canciones que ellas han escrito.
\end{abstract}

Palabras clave: hip hop chicana, xicanismo, ciudad global, barrio postindustrial, barriocentrismo, migración mexicana.

\section{Recibido: 7 de septiembre, evaluado: 18 de noviembre, aprobado: 22 de noviembre.}

1 Maestra en Estudios Culturales (con énfasis en Estudios de Género) del Colegio de la Frontera Norte, México. Traductora, docente e investigadora del Centro de Educación para el Desarrollo, Sede Principal, UNIMINUTO.Correo electrónico: diankaschoen@gmail.com

2 Artículo de investigación derivado de la tesis para optar por el título de Maestría, titulada Guerrera beats: hip hop chicana en los ángeles. Sobre los discursos de lo femenino y las dinámicas de su resistencia y reproducción. Este texto es una revisión extendida del capítulo titulado "Chicana Hip Hop: Expanding Knowledge in the L.A. Barrio" publicado por primera vez en el libro La verdad: An International Dialogue on Hip Hop Latinidades, 2016. 


\begin{abstract}
This paper aims at bringing us closer to the Chicana hip hop world in the city of Los Angeles. It ilustrates the motivations, strategies, and struggles of chicanas who find their beat through rapping. First, there is a discussion on Chicano hip hop in Los Angeles, how it became relevant for the youth growing up in the postindustrial barrio and the importance of the female production as the missing element for a representation of such experience as a whole; second, it deepens into the conflicts these women live and the strategies they develop in this cultural movement; ultimately, there is a discussion on Chicana hip hop as activism. The arguments throughout the text find support in the testimonies gathered through interviews done to five chicana rappers and some fragments of their songs.
\end{abstract}

Keywords: Chicana Hip Hop, Xicanismo, Global City, Postindustrial Barrio, Barriocentrism, Mexican Migration.

\title{
Resumo
}

O presente artigo de investigação realiza uma aproximação ao mundo do hip hop chicana na cidade de Los Angeles para ilustrar as motivações, estratégias e lutas das mulheres que encontram sua bater particularmente no rap. Em primeiro lugar, apresenta-se uma discussão sobre o hip hop chicano na cidade de Los Angeles, sua relevância para a juventude do bairro postindustrial e a importância da produção feminina como elemento faltante para uma representação mais integral dessa experiência. Em segundo lugar, aprofunda-se nos conflitos que estas mulheres experimentam e as estratégias que desenvolvem dentro deste movimento cultural. Finalmente, propõe-se a discussão do hip hop chicana como ativismo. Os argumentos aqui apresentados vêm acompanhados dos depoimentos coletados nas entrevistas feitas a cinco raperas chicanas e alguns fragmentos das letras das canções que elas têm escrito.

Palavras-chave: hip hop chicana, xicanismo, cidade global, bairro postindustrial, barriocentrismo, migração mexicana. 


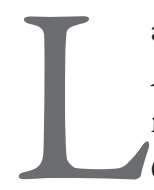

a población latina en el condado de Los Ángeles es un poco mayor a los cinco millones de personas, entre los cuales el $66 \%$ tiene raíces mexicanas. El hip hop en esta área es bastante popular y la producción proveniente de este grupo ha crecido exponencialmente desde los años noventa, momento en que este movimiento se hizo global y diferentes subjetividades se apropiaron de él. Esta apropiación diversificó los estilos de este lenguaje. Este artículo presenta, entonces, una faceta de esta producción, específicamente el lado femenino que canaliza su expresión a través del micrófono.

Las raperas de raíces mexicanas en la comunidad angelina han representado el movimiento desde sus inicios; sin embargo, no se ha documentado de la misma manera que la producción masculina. De hecho, el interés por estudiar el hip hop latino en Estados Unidos es algo reciente. Si bien se ha reconocido la influencia de la comunidad boricua en el desarrollo del breakdancing, por ejemplo, o se ha evidenciado la participación de grafiteros chicanos en el movimiento, los estudios sobre hip hop más bien se han concentrado en la experiencia de la diáspora africana en este país.

Las raperas chicanas como sujeto colectivo representan la complejidad del entramado contextual y simbólico de crecer chicanas en una ciudad global de Estados Unidos con altos contrastes socioculturales. Sus autobiografías, reclamos y críticas en sus canciones revelan tensiones cotidianas de triple opresión por clase, etnia y género; al mismo tiempo que remiten a los conflictos y diferencias que surgen entre las generaciones familiares. Además de esto, su trabajo no sólo permite tener un retrato de las condiciones del barrio, sino que hace posible conocer los contenidos de una lucha compartida: la reivindicación de lo femenino como un lugar de poder desde donde enunciar sus diferencias y negociar las distintas relaciones sociales que las constituye en términos de subordinación.
En este marco, y a lo largo de este artículo, se trata el concepto de hip hop chicana - o chicana hip hop en inglés - como un aporte tanto a los estudios sobre este movimiento cultural como al feminismo de la tercera ola. Para ello es necesario aclarar que, en cuanto al término 'chicana', se usa la definición que Norma Alarcón (1998) ofrece y que significa el "[...] nombre de la resistencia que posibilita puntos de partida y de pensamiento culturales y políticos a través de las múltiples migraciones y dislocaciones de mujeres de ascendencia 'mexicana"' (p. 374). Desde este término se busca identificar la conciencia crítica de saberse un cuerpo historizado con múltiples construcciones étnico-raciales que lo significan principalmente; pero también sexuales y de género - si se reconoce el aporte del xicanismo ${ }^{3}$ en la materia-.

De este modo, el hip hop chicana se define como un foro dialógico para producir una presencia femenina poderosa que interviene creativamente en el ordenamiento del mundo mediante las formas artísticas del hip hop y desde donde se reconoce al barrio postindustrial como punto de partida en la articulación de experiencias de vida, ideas, deseos, opiniones, críticas y luchas que representan las múltiples migraciones y dislocaciones de mujeres de ascendencia mexicana en Estados Unidos. Su producción puede ser en inglés, en español o en spanglish, lo que evidencia la multiplicidad de códigos que llevan encima y que asumen como propios. Cada idioma lleva intrínsecas unas relaciones de poder históricas, territoriales y socioculturales, lo cual hace que posicionarse en uno u otro sea un auto-reconocimiento y esté profundamente relacionado con la identidad (Espín, 1999).

El presente artículo busca ilustrar las motivaciones, estrategias y luchas del hip hop chicana de la ciudad de Los Ángeles que encuentra su latir en el rap. Las reflexiones acá expuestas son el resultado de un trabajo de investigación cualitativa que tomó lugar entre 2010 y 2012 para obtener el grado de Maestra en Estudios Culturales. Los criterios

3 El xicanismo busca evidenciar que la identidad étnica y de género es histórica y no biológica. Además, posiciona lo indígena como el centro mismo del proceso de identificación, como posicionamiento políico para descolonizar saberes y discursos. 
de selección de las artistas para la investigación tuvieron dos dimensiones: a) homogénea; ser mujeres adultas, chicanas, raperas y que hayan crecido en el condado de Los Ángeles, que hablen español y que tengan su producción artística grabada y audible en la red; b) heterogénea: que pertenezcan a distintas generaciones de migrantes mexicanos y que representen diferentes estilos de rap.

La importancia del bilingüismo en el grupo parte del hecho de que la evocación de memorias y la generación de opiniones, críticas o reflexiones están cargadas del contexto lingüístico en el que se vivió la experiencia. De este modo, se escogieron cinco participantes para conformar este estudio a nivel micro: Tenochtitlán, Eyerie y Centzi del grupo Guerrilla Queenz, Cihuatl Ce y Fe.

La técnica principal de recolección de datos fue la de relatos de vida, con los que se indagó en torno a aspectos significativos de la constitución femenina, historia migratoria y experiencias dentro del hip hop angelino. Los recuerdos, visión propia de los acontecimientos y reflexiones retrospectivas de los hechos comprenden una matriz de significados que al ser editados por la relatora misma se construyen como los más importantes para ella, los que más dotaron de sentido su realidad en esos momentos. La observación participante y el análisis de las letras de sus canciones también apoyaron la investigación.

Los argumentos presentados en este artículo vienen acompañados de los testimonios recolectados en las entrevistas y algunos fragmentos de las letras de las canciones que estas cinco mujeres han escrito. Así las cosas, primero se presenta una discusión sobre el hip hop chicano en la ciudad de Los Ángeles, su relevancia para la juventud del barrio postindustrial y la importancia de la producción femenina como el elemento faltante para una representación más integral de esa experiencia. En segundo lugar, se profundiza en los conflictos que experimentan y las estrategias que estas mujeres desarrollan dentro del movimiento. Finalmente, se plantea la discusión del chicana hip hop como activismo.

\section{Chicano beats en Lost Ángeles}

\author{
Don't take my block lightly \\ We're screwed up like everybody in the world might be \\ Born into Union and 17th \\ Out of the womb in the 70's \\ Repping Lost Angeles through musical melodies.
}

(SICK JACKEN, 2009)

La era postindustrial en Estados Unidos se desarrolla en los años setenta y madura con las reformas neoliberales implementadas a partir del periodo de Ronald Reagan. Durante todo el proceso, los centros urbanos han reflejado un complejo conjunto de fuerzas globales nunca antes experimentadas que han contribuido a la reestructuración económica y social de las ciudades estadounidenses: el crecimiento de redes de telecomunicación multinacionales, la competencia económica en dimensiones globales, una revolución tecnológica sin precedentes, la formación de una nueva división internacional del trabajo, el incremento de poder relativo a las finanzas y nuevos patrones migratorios provenientes de los países en desarrollo (Rose, 1994).

Pero dicho proceso de globalización produce efectos contrarios en la experiencia humana del tiempo/espacio. Mientras que para algunos es un proceso de fluidez, simultaneidad, velocidad y liberación del espacio, para otros es de estancamiento, lentitud y localización (Bauman, 2001, p. 28). En dicha polarización, “[...] las redes locales, fragmentadas, con frecuencia definidas étnicamente, utilizan su identidad como el recurso más precioso para defender sus intereses y hasta su propia existencia" (Castells, 1989, p. 228).

Para los años ochenta, gracias al flujo de capital japonés y canadiense, la ciudad de Los Ángeles se convirtió en el segundo polo financiero del Círculo del Pacífico, pues, después de Tokio, la ciudad comenzó a exhibir su poder sobre el espacio geográfico con monumentos emblemáticos que la convertían en toda una ciudad global (Davis, 1990). 
Sin embargo, en las llamadas inner-cities se implementaban drásticos recortes en los recursos públicos. Ana Castillo señala que todos los logros alcanzados por el movimiento chicano/latino quedaron prácticamente ahogados, pues el colectivismo y la búsqueda por mantener la cultura mexica amerindia con proyectos comunitarios, de rehabilitación, educación alternativa, consejerías juveniles, refugios para mujeres, etc. eran financiados por el gobierno (Rose, 1994). De esta manera, con la reconfiguración económica, los espacios que habitaban las comunidades mexicanas y chicanas se convirtieron en barrios postindustriales cuyas características principales son: "el aumento en los niveles de pobreza, encarcelamiento, violencia, drogas y pandillas; también el desempleo, la falta de acceso a buenas escuelas y programas de servicio social" (McFarland, 2008, p. 22).

En estos barrios, las diásporas africana y latina, especialmente mexicana y centroamericana, han estado en constante interacción. Han compartido desde los mismos espacios de vivienda y laborales hasta las luchas de los derechos civiles y el activismo sindical (Johnson, 2002, p. 316). En las calles y las escuelas de los barrios postindustriales o guetos, los jóvenes comparten conocimientos y prácticas que los identifica como parte de una matriz cultural que los racializa y segrega, pero que a la vez les ofrece la posibilidad de resistir y desafiar creativamente tanto los prejuicios raciales como los estereotipos que los devalúan y desempoderan; sin embargo, este complejo intercambio cultural no se da en un espacio libre de conflictos, al contrario, la tensión cotidiana entre estas dos comunidades comprende altos niveles de hostilidad como resultado de un conjunto de factores que se resume en la competencia por los escasos recursos que tienen y la aceptación acrítica de los estereotipos racistas impuestos por la cultura dominante.
No obstante, en medio del proceso de desindustrialización de finales del Siglo xx, este intercambio cultural da origen a un hip hop chicano cuyo rap viaja sobre beats de funk y soul, y un hip hop afro que se apropia de las calles angelinas con la cultura lowrider ${ }^{4}$ chicana (McFarland, 2006, p. 943).

El hip hop angelino se hizo reconocido mundialmente por ser el lugar de origen del gangsta rap $^{5}$. La propuesta de N.W.A (Niggas with Attitute) y su trabajo Straight Outta Compton en 1988 es una construcción discursiva que se concentra en un barrio específico, lo cual resulta en contraste con la abstracción del gueto Queensbridge/ Bronx/Harlem que se proponía en Nueva York. 'The Hood' o el barrio está construido en términos de 'home' o el hogar, y representar el barrio es construir su territorio y las situaciones escenas y sitios que estén allí contenidos en la vida cotidiana. En la práctica las letras y rimas del artista deben obtener el reconocimiento de los habitantes del barrio; éstos deben poder reconocerse en esa experiencia; el estilo y las imágenes deben resonar de manera significativa en ellos para avalarla como verdadera, unas rimas que logren keeping it real (Forman, 2004 , pp. 207-208).

Mi disco cuenta sobre cómo crecí en la ciudad de Los Ángeles. Crecí por la tercera y la Rampart [Rampart District]. Es un área donde hay muchas pandillas y casi todas las noches había helicópteros. A veces toda la noche. [...] But it was really explosive, because you would go down one street and you are in "18 neighborhood". And then you go down one more street and then you are in "MS territory" [Mara Salvatrucha]. So, a lot of the hip hop coming out of this area was Gangsta rap. (Tenochtitlán. Entrevista directa, 2011) ${ }^{6}$.

4 Cultura estética mexicano-americana que desde los años cincuenta estiliza los vehículos (carros, motos y bicicletas) para que rueden a ras del suelo; éstos llevan ilustraciones, colores cromados llamativos y otros elementos que resalten. La juventud de los barrios latinos fueron desarrollando esta estética como reafirmación cultural y política frente a la cultura anglo dominante.

5 Subgénero del rap que nace en Los Ángeles y que relata la cotidianidad del joven pandillero angelino: la vida en las calles como traficador de drogas y de mujeres (proxenitismo) y la violencia intrínseca y explícita de esa vida. El hip hop, y especialmente el rap, se convirtió en el "canal de noticias" de las pandillas en esta ciudad.

6 Tenochtitlán tenía 29 años en el momento de la entrevista. Es madre sola. Su auto-adscripción étnica va desde Mexican-American, a chicana y Latina. Su trabajo musical lo cataloga en el subgénero de Hardcore Underground hip hop. 
El rap en la Costa Oeste tiene su anclaje en las especificidades del entorno inmediato, lo que diferencia un barrio o una calle de otros dentro del mismo gueto, empezando por las pandillas o 'gangs' que se adueñan de calles específicas dentro del mismo barrio. Las pandillas más legendarias en Los Ángeles son The Bloods y The Crips ${ }^{7}$, afroamericanas, y en la población chicana Los Cholos $^{8} \mathrm{de}$ East L.A. cuya lucha por el territorio de la ciudad se hizo cada vez más violenta desde los años ochenta por el control de la distribución transnacional de cocaína y 'crack' (Davis, 1990). También en los ochentas, se conformaron nuevos grupos inscritos en los procesos migratorios de Los Ángeles: la Mara Salvatrucha (MS-13) y el Barrio 18, con hombres y mujeres salvadoreños, guatemaltecos y hondureños y con características parecidas a los cholos mexicanos (Nateras Domínguez, 2007).

Casi una década le tomó al hip hop chicano entrar en la conciencia de la cultura popular nacional, a pesar de que las chicanas y chicanos llevaban haciendo rap, graffitti, d.j. y breakdancing desde que se conformó en el lenguaje del barrio a principios de los años 80. En 1990, el disco Hispanic Causing Panic de Kid Frost y su éxito "La Raza" se convirtió en el estandarte de la experiencia juvenil de la diáspora mexicana en ese periodo. En dicha canción, Frost conjuga el caló, los saberes de la calle del barrio latino, el nacionalismo cultural chicano, una crítica política y una identidad hipermasculina y heterosexual que producen el efecto de formar un locus de características culturales, raciales y de género que resuenan en la audiencia chicana androcentrista de la escena lowrider y chola (Perez-Torres, 2006, p. 326). Esta escena es de las más fortalecidas en la ciudad hasta el día de hoy.

En ese mismo año, Lighter Shade of Brown (LSOB) saca su album Brown and Proud y un año después Cypress Hill publica su trabajo homónimo, lo cual provoca una ola de grupos localizados en el barrio postindustrial angelino que entraron al mercado mainstream norteamericano como Psycho Realm y Delinquent Habits. Cypress Hill, a diferencia de Frost, no ofrece un discurso nacionalista, sino barriocéntrico; un discurso que evidencia que las raíces urbanas de las clases bajas [the underclass urban roots] son multiétnicas y muestran un orgullo ante ello:

Cypress Hill reflects the realities of inner-city life for many people of color as one of cultural exchange, borrowing, transformation, and common struggle. They as well as Chicano and other rappers, appeal to our youth of color because they acknowledge a multiethnic, multiracial, post-industrial, inner-city experience that political leaders from an older generation rarely engage. (McFarland, 2006, p. 951).

La diversidad del hip hop angelino se expande según las necesidades de representación de sus habitantes. Cansados de la propuesta gangsta, cuya misoginia, violencia y lenguaje agresivo dejaba por fuera mucho de la experiencia de ser un joven de color en el barrio; se conforma a inicios de los noventa el café The Good Life, en Crenshaw, el cual ofreció su espacio todos los jueves para que los jóvenes pudieran desarrollar sus talentos creativos, especialmente en el Freestyle. Así nació Project Blowed, un espacio para competir y compartir, el más influyente en el desarrollo de la escena subterránea del hip hop angelino. $\mathrm{Y}$ a pesar de que fue un espacio netamente afroamericano, algunos artistas de la diáspora mexicana construyeron sus carreras allí como 2Mex y Olmeca.

When I first went to Project Blowed, I saw the female group Figures of Speech. Those sisters had a song that was all like bashing on the system! You know! like basically talking about decolonization, and this is when I am 17, 18 and holy shit! There is a different hip hop? What is going on! I am like awake to a whole different world I did not know

7 Los Crips simbolizan su pertenencia al grupo con el color azul y los Bloods con el color rojo. El uso de los colores patrióticos como identificación significaba que quien entrara con los colores incorrectos a territorio azul o rojo podría ser asesinado (Morgan, 2009). Varios raperos reconocidos hoy mundialmente provienen de esas pandillas: Snoop Dog y Ice-T son Crips y B-Real y Sen Dog de Cypress Hill son Bloods.

8 Los Cholos son una cultura juvenil fronteriza (Mex/Eevu) que desarrollaron una gestualidad y corporalidad inconfundible, una de sus principales características es la de marcar todo su cuerpo con tatuajes y representar con las manos y los brazos las iniciales del barrio al que se pertenece —las Maras años después adoptan también esa estética - (Valenzuela, 2007, p. 55). 
about! That is, to me, "Decolonizing Hip Hop". (Eyerie, Entrevista directa, 2012).

$\mathrm{Al}$ hacer un rastreo de las mujeres más representativas del hip hop femenino en esta ciudad, el grupo Figures of Speech y la artista Medusa son las más destacadas. Ambas propuestas maduraron en Project Blowed y son de mujeres afroamericanas. $\mathrm{Al}$ indagar por artistas femeninas chicanas de los años noventa, ningún nombre sale a flote, pues si bien existen no llegaron a hacer parte activa de la escena local hasta que los sellos independientes comenzaron a representarlas entrado el siglo XXI o ellas mismas produjeron sus trabajos y comenzaron a participar más activamente en el movimiento.

A inicios de este siglo la diversidad del hip hop chicano ha tomado varios matices, entre esos está la mezcla explícita de música tradicional mexicana como los corridos o música de banda. Entre los más reconocidos está Akwid, el grupo de dos mexicanos que crecieron en Los Ángeles y que llegaron a ganar un Grammy y dos premios Billboard. También madura la propuesta indigenista, como la de El Vuh, una que se relaciona más con el xicanismo, el cual exalta y enfatiza las raíces y la espiritualidad indígenas, al igual que se discuten temáticas políticas de la lucha indígena (McFarland, 2008, p. 55).

El académico del hip hop Pancho McFarland ha realizado un riguroso estudio de este movimiento, especialmente del rap chicano. Su análisis va desde la apropiación y transformación del hip hop por parte de los jóvenes chicanos y mexicanos en las ciudades del sur de los Estados Unidos al identificar sus diferentes vertientes en la lucha por la auto-determinación; hasta un profundo análisis de su relación con el territorio, la historia, la violencia, sus recursos y estrategias e incluso las ideologías de género que permean sus políticas de representación. Él reconoce que el hip hop chicano ha desarrollado una epistemología y ontología única a través de la experiencia vivida en las calles del barrio, "an experience of dislocation and empowerment, ciminalization and liberation" (McFarland, 2013, p. 32).

En su estudio llamado Chicano Rap. Gender and Violence in the Postindustrial Barrio, MacFarland (2008) encontró que la objetivación de la mujer y la violencia son temas recurrentes de manifestación de poder y señala que son de tres tipos los productos culturales que contribuyen a este fenómeno: los medios masivos dominantes controlados por grandes corporaciones que naturalizan valores androcentristas y etno-reduccionistas/ estereotipos; los aspectos de la hombría afroamericana - de la cual se ha influenciado y que ha sido altamente mercadeada en el cine-; y la cultura patriarcal mexicana y chicana. Esta última con un discurso binario que reduce el papel de la mujer a virgen/puta, dicotomía que encuentra terreno fértil en el rap chicano. Estas representaciones de la feminidad son uni-dimensionales y fracasan al captar o valorar la complejidad femenina. En las canciones de rap masculino, las dos posibilidades de ser mujer se resumen en el binario de reproducción/erotismo y esto ocurre tanto en el rap chicano cholo como en el barriocéntrico pluriétnico.

En el caso del hip hop indigenista o xicanista, su aporte va un poco más lejos, pues busca empoderar al sujeto femenino, solo que se queda en resaltar las virtudes femeninas desde la reproducción y su poder de cohesionar la comunidad; sin embargo, en cuanto a abrirles espacios para desarrollarse como intelectuales hip hop al igual que los hombres, colaborar en sus producciones canciones o invitarlas como artistas a conciertos, las relaciones siguen siendo excluyentes ${ }^{9}$. La raíz patriarcal heteronormativa es tan profunda, que las reflexiones al

9 El xicanismo (con equis) plantea que la identidad xicana es historizada y construida desde múltiples construcciones raciales sobre los cuerpos desde "el descubrimiento" de los pueblos originales en América [el continente]. Ser xicano es reconocerse en un cuerpo donde se intersectan distintos niveles 0 dimensiones: género, clase, sexualidad, etnicidad; todos igual de importantes. El xicanismo se diferencia del chicanismo porque mientras el segundo se concentraba en una experiencia mexico-americana, el primero es elástico y acepta todo tipo de raíces — Centro y Suramérica—; también busca conectarse con los movimientos indígenas de todo el mundo (Ríos, 2005). El uso de la Xes " . . . ] lo que Ana Castillo llama la 'poética' de la Xicanista, la cual corresponde a una nueva noción de color e identidad que involucra una revisión de las teorías monolíticas de mestizaje y la hibridez racial para re-evaluar a la mujer indígena y situarla en el puro centro de su identidad racial" (Velasco, 2006, p. 208); es decir, en el centro simbólico de la comunidad imaginada identitaria. 
respecto tomarán mucho tiempo. A pesar de que el hip hop indígena/mexica provee una retórica fina de resistencia y crítica al capitalismo, colonialismo y racismo:

Their claims to developing a more equitable society with superior nature-society relations will fall short as women and their ideas are marginalized and made into passive spectators of males warrior action. The patriarchal heteronormative understanding of the world, and especially of gender, severely limits the possibilities that the neo-indigenist cultural and political movement can lead to a more equitable, open, and just world. (McFarland, 2013, p. 79).

Pero como es de comprender, a quienes les corresponde producir una presencia femenina compleja y poderosa en el hip hop es a las mujeres. Usualmente, las mujeres tienden a insertarse en las grupalidades juveniles 'invisibilizando' sus 'diferencias' (Reguillo, 2003, p. 375); no obstante, aunque consideradas minorías, siempre hay mujeres que se resisten a ser invisibilizadas y representadas, por lo cual se lanzan a ofrecer creativamente su visión del mundo. A continuación, se desarrolla una interpretación genérica sobre la participación de las chicanas en el rap angelino. En dicho análisis se presentan sus luchas dentro del movimiento local, sus estrategias de empoderamiento y el uso práctico que le dan a su obra, lo cual nos permite ver una fotografía más completa del barrio postindustrial angelino.

\section{Guerrera beats: hip hop chicana y sus saberes en el barrio}

Desde la reubicación de los límites fronterizos entre Estados Unidos y México en el siglo XIX, la migración mexicana ha sido una constante y ha cambiado su naturaleza con las políticas entre los dos países y los cambios históricos. Hasta 1980, el flujo proveniente de México era principalmente masculino, temporal y de origen rural (Massey et al., 1987). Desde los años ochenta, estudios han encontrado que la migración es ahora indocumentada, toda la familia está involucrada y los flujos se han feminizado (Leite, Angoa, \& Rodríguez, 2009).
La reconfiguración económica ha tenido impactos diferenciados "[...] en los hombres y en las mujeres, en las culturas laborales femeninas y masculinas, y en las formas de poder y empoderamiento femenino y masculino" (Sassen, 2005, p. 110). Si bien los hombres de color e inmigrantes han visto disminuidas sus oportunidades laborales en los nichos primarios que para los latinos son normalmente la jardinería, la preparación de alimentos y la construcción (Davis, 2000, p. 96). Las mujeres inmigrantes han visto amplificado su espectro laboral, pues representan mano de obra aún más barata que la masculina y ha habido un crecimiento de circuitos globales alternativos.

Las mujeres lloran

Dejando sus niños en el otro lado

Trabajando como esclavas día y noche

Por un peso, que ya no vale nada

Están, estamos, en la calle pidiendo, vendiendo

Esperando.... Y ahora organizando

Tratando, no de sobrevivir

Pero de vivir!

Pero nos están atacando de diferentes lados

Por las cuatro direcciones sagradas

Necesitamos defendernos

En el norte Juárez - mis hermanas, las mujeres de las maquiladoras

Por el sur, sureños peleando con los de la otra calle

Eastside - Westside

Somos de la misma tribu

Una nación debajo de la represión, de la policía, de la migra...

(Fe, 2010, “The Women Weep”).

En esta canción Fe identifica este sistema de explotación en el que está sumida la realidad femenina. Ella reconoce no sólo los sacrificios de la mujer migrante y lo poco valorada que se encuentra en su sociedad, pero también las relaciones de dominación que se ejerce sobre el cuerpo femenino a uno 
y otro lado de la frontera. Por lo tanto, ella invita a la unión y a reconocer la fuerza de estas mujeres y de la comunidad misma. Ella logra retratar lo que Sassen (2000) describe como la era de la "feminización de la supervivencia”, donde tanto los hogares, como las comunidades y hasta los gobiernos están cada vez más dependiendo de las mujeres para sobrevivir (p. 520). Es un sistema donde la mujer migrante indocumentada representa "[...] una ventaja para el empleador, pues pueden explotar los valores de subordinación que han sido incorporados socialmente" (Kittay, 2010, p. 62).

En el caso de las mujeres chicanas en Estado Unidos, ellas se encuentran entre los grupos más excluidos con los ingresos más bajos, menos posibilidades de acceso a la educación y son víctimas de constante discriminación racial. Ante esta problemática de triple opresión en que las mujeres chicanas se construyen, algunas de ellas encuentran en el rap una manera para expresarse y muchas veces articular creativamente posibilidades de liberación.

De todas las otras formas estéticas del hip hop ellas escogen el rap porque el valor que obtiene está en la calidad lírica, poética, la veracidad de la historia y la habilidad de crear nuevas palabras, "[...] hip hop is great storytelling, and it's all about how we grew up in the streets. There's a lot of wordplay, it's very clever, there's humor, there's really dry humor and I love it!" (Tenochtitlán. Entrevista directa, 2012). En cuanto a la música que lo acompaña, el poder del beat es el que canaliza la conexión con las emociones que producen la identificación con el lugar, el territorio: sea el barrio, una calle específica, la ciudad, la pachamama.

Hip Hop is definitely alter-native; it has that native way wherever it's coming from. Understanding the indigenous beat, the heartbeat, the tambor that cultures come from, wherever you come from in the world, there is that indigenous ancestry, there's a beat, usually. There's a heartbeat, and youth are connected to that, feel that, and move to it. (Fe, Entrevista directa, 2012) ${ }^{10}$.
Simon Frith (2003) señala que “[...] el rap es una forma basada en la voz con un sentido de la presencia excepcionalmente fuerte" (p. 194) y que al ser música, ofrece con intensidad una percepción del yo, como de los otros, de los subjetivo en lo colectivo. El rap "[...] es identidad producida en la ejecución” (p. 194); es un medio estético para producir presencia, una que reconoce el lugar en el que se encuentra y desde donde se articulan luchas por el derecho a estar ahí.

Las temáticas de sus canciones varían según las emociones y pensamientos que tenga el artista para compartir. Normalmente, las historias están cargadas de frustración, trauma, dolor, pero también esperanza, buen humor y unidad comunal. Algo que no falta en la transmisión del mensaje es dejar en claro desde dónde se está hablando, por lo cual se hace explícito el lugar y la comunidad imaginada que se está representando: Costa este, Costa oeste, Los Ángeles, algún distrito específico, barrio, pandilla, afro, chicano, cholo, indígena, etcétera; y describir las condiciones de opresión que desde una mirada de subalternidad allí se viven.

Para intervenir creativamente en el ordenamiento del mundo en un movimiento cultural y musical donde predomina la ejecución de una identidad masculina que ha establecido una matriz lógica donde se define el talento de un artista por la capacidad que tiene un individuo de get the crowd hyped o emocionar y agitar al público; esto no es algo sencillo para un sujeto femenino. Al ser un hip hop masculino, el público también lo es y las mujeres, normalmente no participan activamente en el movimiento, sino como acompañantes de los hombres. Es decir, el hip hop ha sido una plataforma para re-producir una presencia poderosa masculina y el talento depende del grado de identificación que el colectivo encuentre en el discurso dado y el estilo que use en simultáneo con el goce que se sienta al ritmo del beat.

The industry is male dominated and male supportive. When you look at a female you look at sex, you look

10 Fe tenía 35 años cuando realizó la entrevista. No tiene hijos y vive en Unión libre con su pareja. Su autoadscripción étnica es Xicana 0 chicana indígena. Su producción musical la Ilama Floetry Hip Hop. 
at sells, of objectifying before you make a credible choice about their talent. For men is not like that. They are very supportive of each other's music, put a lot of shows, brotherhood and solidarity. So it is a cause to be motivated to do what we do. It pushes me to be the best I can in everything that I do, my music, my poems... (Eyerie. Entrevista directa, 2012) $)^{11}$.

Esa es precisamente la lucha constante a la que están enfrentadas las mujeres que deciden entrar a este juego, a que se les reconozca como artistas, donde el talento sea una cualidad humana, más allá de una capacidad masculina o femenina. Un aspecto interesante en el tipo de actitudes o energías transmitidas por mujeres, como manera inconsciente de dirigir su talento, es la agresividad, la cual ha sido relacionada históricamente con lo masculino.

But also, I think that all of us here, as women, get really aggressive on the mic. It's very rare that we do a really soft song. It is almost kinda as to prove our skill level or that we can do it just as good as men, we tend to get aggressive on the mic. (Cihuatl Ce. Entrevista directa, 2012) ${ }^{12}$.

Si las expresiones dominantes en el hip hop son masculinas y dentro de su matriz de significados es donde están los términos para competir y diferenciar el talento de la mediocridad, las mujeres que entran en un campo de este tipo asumen los contenidos de la masculinidad no sólo para poder competir, sino porque tiene sentido para ellas. Desde su humanidad integral, ellas también sienten frustración, rabia y dolor sobre lo que experimentan en su ciudad, sus calles, sus familias, sus cuerpos; y por ende, han tenido que desarrollar actitudes agresivas para posicionarse asertivamente en el poder. El hip hop les da ese canal para estar ahí de esa manera. Al hacerlo, ellas desplazan los sentidos naturalizados de lo femenino exhibiéndolos como un artificio, una arbitrariedad, moviendo las fronteras más hacia un balance en la producción de conocimiento creativo sobre la realidad de la ciudad global desde una perspectiva femenina.

We're going to speak about our reality, we're going to speak our lives, we're going to speak about our truths, what affects us! And what affects us as women is different from what affects us as men. A male might talk about a domestic violence song, they might! - a few of them have! - pero siempre va a ser desde la perspective of a male, not a perspective of a female. So in that sense is where I see the difference. (Centzi, Entrevista directa, 2012) ${ }^{13}$.

Para las cinco participantes, no sólo la violencia que ejerce la policía contra los habitantes del barrio y la producida por el crimen organizado invaden la vida cotidiana, sino también la violencia contra la mujer es una práctica constante en las familias o en las calles del barrio. Muchos de estos abusos se viven en silencio y muchos quedan impunes, pues nadie se arriesga a inculpar a nadie con la policía, además de que también se han conocido casos de abusos por parte de ésta contra mujeres latinas.

She was only 24 when she was found face down on the beach

dead from a beat down

No one but the waves still hear the sound of the scream of her pain

And it's going on all around

Tears falling like the rain $[\ldots]$

And she was only 24

Possibilities should be explored, 'cause see... we' re either bitches or whores

Our life is disposable! Casualties of war!

11 Eyerie tenía 32 años cuando se realizó la entrevista. Es madre sola y su autoadscripción étnica es afromexicana o blaxican. Es rapera de Guerrilla Queenz, grupo de Revolutionary Indigenous Hip Hop.

12 Cihuatl Ce tenía 32 años cuando se realizó la entrevista. Es madre sola. Su autoadscripción étnica es mexicana 0 rrban indigenous. Su producción musical la cataloga dentro del término Indigenous Hip Hop.

13 Centzi tenía 33 años cuando se realizó la entrevista. Es madre de cuatro hijos y vive con su compañero. Es rapera en Guerrilla Queenz, grupo de Revolutionary Indigenous Hip Hop. 
How many females would be ignored!

How many funerals! How many burials!

Before the hardcore man and women warriors begin a dialogue!

Creating protocols...

'cause when our women die, we all fall.

So stand tall, listen to the call, listen to the scream, listen to the cries!

Don't let another Brown sister die!

(Cihuatl Ce, 2010, "Not another ever again!").

Como lo expresa Cihuatl Ce en esta canción parece no haber valor positivo que signifique lo femenino en esta sociedad. El irrespeto está encerrado en los significantes que cautivan a la mujer en objeto sexual, lo cual después de usada la hace desechable. Crecer chicana en el barrio es construirse dentro de ideales tradicionales de la mujer mexicana transmitidos por la familia, ideales femeninos del grupo dominante que se transmiten por los medios, la influencia del discurso feminista de clase media y de color (o segunda y tercera ola del feminismo) y la realidad que experimentan en su vida cotidiana.

Crecer y hacerse chicana en estas condiciones de devaluación requiere de enfrentarse continuamente a esa uni-dimensionalidad que ciñe a los cuerpos femeninos a ser mediadores 'naturales' de satisfacción para otros y conducir una búsqueda interminable por la re-edición de esos términos, por la negociación de su representación. Por esta razón, ellas hacen una revisión consciente de los símbolos que comparten en su entorno y cómo estos se traducen en la significación de sus cuerpos como sujetos devaluados.

En la sociedad actual, la mujer ha sido desvalorizada, no se le tiene respeto, y está objetivizada sexualmente o sólo es alguien que está para el servicio de otros. Hay que devolverle lo sagrado a la mujer. Por eso ella también no es solo creadora/madre sino guerrera, siempre luchando por su familia, su comunidad, su futuro. Es una mujer que tiene auto-empowerment, que tiene poder de sí misma, que tiene el poder por dentro, que no necesita validación de cualquier otra persona porque esa mujer tiene su valor interno ${ }_{i} \mathrm{No}$ se lo pueden robar! (Eyerie. Entrevista directa, 2012).

En el discurso de todas las raperas entrevistadas hay un acuerdo claro sobre la devaluación de lo femenino y el irrespeto que rodea esta subestimación en la sociedad en la que viven, por la historia que han heredado, pero también un deseo común en transformarlo. Ellas se remiten a su historia indígena para encontrar arquetipos femeninos de poder y deconstruir aquellos tradicionales sin soberanía y trascendencia.

En este ejercicio hasta buscan llegar a una memoria prepatriarcal de la misma, si la hay. Este camino les ofrece la posibilidad de descolonizar los significados que han inscrito y disciplinado sus cuerpos y los ha objetivizado al nivel de la explotación hasta el desecho. Los arquetipos de diosas poderosas las alivia de la 'impotencia natural' y les ofrece otros términos con qué renegociar su condición histórica de subordinación. Es así que la recuperación y apropiación de lo femenino desde la gran Diosa Madre — en oposición a la Madre de Dios- entra en juego ${ }^{14}$. De esta manera, se presenta a la mujer en una posición sagrada, asociada a la madre tierra y se destaca su poder creador, pero también destructor.

A temple statue hits the ground. It cracks and crumbles.

It is la Diosa Azteca, I emerge from the rubble

Awakened from ages of a deep state of slumber.

14 En la cosmogonía de los Toltecas, Coatlicue, la de la falda de serpientes, era la Gran Diosa Madre que contenía y balanceaba las dualidades de lo femenino y masculino, la luz y la oscuridad, la vida y la muerte, la creación y la destrucción. En esos tiempos, las mujeres podían poseer propiedades, curaban y eran sacerdotisas. La pérdida del balance entre oposiciones comienza luego del peregrinaje a Aztlán, con el tiempo adoptan a Tetzauhteotl Huitzilopochtli, dios del sol, como el gran dios de su sistema religioso y el poder de Coatlicue quedó reducido a ser la madre de este dios (Anzaldúa, 1990, pp. 54-56), conformando así el arquetipo femenino androcéntrico mexica donde se venera a la madre, pero subordinada al hombre. Este arquetipo viene a confirmarse con la colonización y la imposición católica de la Virgen como la madre de Dios. 
Drop, hit the deck, seek refuge, take cover.

I'm more deadly vicious than a pack of velociraptor.

With the target on-lock, and it's the rap game I'm after.

I spit acid that causes lethal, mental erosion.

Your dumb-founded can't take it, causing cerebral implosion.

Like ceremonial skulls, I put dunces on skewers.

Pseudo-hip hop's the plague,

Fear not, cause Teno's the cure!

(Tenochtitlan, 2010, “1519”).

La Diosa Madre, creadora y quitadora de vida, nutridora y terrible, dueña de su cuerpo y su ejercicio sexual, todapoderosa, autónoma y soberana (Maier, 2001), les ofrece una gama de términos que dotan de sentido su realidad de mujeres en el barrio postindustrial de una manera más coherente que el de la Madre de Dios. Además, les ofrece una dimensión espiritual distinta a la experimentada en la religión católica, porque buscan conectarse con la dualidad interna y universal con miras a conocerse y equilibrar dicha dualidad. De este modo, términos como guerrera, en balance, independiente y creadora conforman las cualidades de la mujer ideal de estas raperas.

\section{Hip hop chicana como activismo}

I see hip hop as a driving force within the youth throughout the whole world to express their reality of what's going on! Hip hop is very effective. So that's why we entered into all this hip hop world, because there is power behind hip hop. Capitalism took it over. Look at what it is now! They reproduce an idea that best gives them money. But the root is there! It's still talking. It's still exists in the underground! We have to use those media as well to push it in! There's been a rise of Hip Hop being used as activism as well. (Centzi, Entrevista directa, 2012).

En este comentario de Centzi hay varios elementos que se deben rescatar y que deben ser explicados para entender la complejidad del fenómeno del hip hop en la ciudad global de Los Ángeles. En primer lugar, ella habla del poder del hip hop como un medio estético que capta la atención de la gente y con el que se puede competir en la disputa por los significados que toma lugar en la ciudad. Reconocer esta fuerza la impulsa a dirigir sus intereses por este medio. En segundo lugar, ella expone que dicho poder también ha sido reconocido por el capitalismo - como ella lo refiere- y por lo tanto se ha apoderado de él y ha privilegiado los discursos de consumo, violencia y sexo como elementos que más se traducen en dinero, sobre los discursos de crítica social y unidad comunitaria. Pero, como explica ella, las y los jóvenes del gueto, del barrio, siguen representando sus experiencias de las maneras que más sentido tienen para ellos y van acorde con sus realidades. De este modo, se puede dar una breve y sencilla manera de entender la diferencia entre el hip hop mainstream y el hip hop underground. Todas las participantes se encontrarían dentro de este último grupo.

Ellas también reconocen dos maneras de posicionarse dentro del underground: como relator de realidades o storytelling, o como quien relata pero también actúa en la comunidad para generar un cambio. Dentro de esta división se podría afirmar que las cinco participantes hacen parte de esa última propuesta, pues su interés principal no es comercial, sino el de reunir fuerzas para la transformación. "When you want to get heard in the streets you have to use the vernacular of the streets and in order to do so, that includes a lot of hip hop!" (Eyerie. Entrevista directa, 2012).

La forma que toma su activismo se concentra en dos niveles: en lo objetivo y en lo simbólico. En el primero, según las entrevistadas, éste se reconoce como emprendimiento comunitario y trata de innovar en ideas y acciones que provengan de la comunidad misma para su desarrollo. Estas comunidades tienen claro que depender de los recursos gubernamentales es algo momentáneo, porque apenas surja una coyuntura económica los primeros recortes en el presupuesto se reflejan en sus barrios. Este aspecto del activismo se encontró como crucial en las razones por las cuales estas chicas se sintieron atraídas por él, pues es una forma de ser activo en la comunidad, en la ciudad. 
Artists in hip hop could pay more attention to problems in the community. There are some things that we cannot fix. But there's a lot of other things that we can. We don't need the big bureaucracy or D.C. it's our neighborhood, it's our home! That's why I put together the Hip Hop for Humanity Food Drive. It was a very simple idea that anyone can do! (Tenochtitlán. Entrevista directa, 2012).

Tenochtitlán, por ejemplo, tiene claro que los problemas de su barrio no los solucionará, ni ha solucionado la gran burocracia distrital y por lo tanto hay que organizarse para recoger los colchones que se quedan tirados en las calles por meses, recoger la basura, pintar las calles, etcétera. Ella ve que en el underground hip hop los artistas hablan sobre las grandes dificultades que fue crecer en sus barrios, pero que no actúan para que mejoren de alguna manera y que los niños y niñas que allí están creciendo puedan tener una oportunidad ${ }^{15}$.

La otra forma de hacer un hip hop como activismo es canalizando los mensajes del rap mismo para no sólo ofrecer descripciones y relatos de las dificultades de vivir en el barrio postindustrial, sino también para influir en el estado de las cosas mediante las reflexiones que éstas han provocado en el sujeto y que invitan a imaginar una comunidad en condiciones de dignidad. Lo que las cinco mujeres entrevistadas comparten es que su acción en el rap se puede traducir en su forma de ejercer poder para la transformación de la historia. Las maneras en que lo hacen tienen dos componentes: descolonizar y curar. Prácticamente, el primero es una búsqueda por desmantelar las nociones biologicistas, ahistóricas o esencialistas con la que los discursos dominantes han construido las diferenciaciones de género, étnicas, de clase y generacional que exponen formas de poder y de opresión históricas que los sitúan en desventaja y en condiciones de no-poder. El segundo tiene como objetivo ofrecer otras significaciones para dotar de nuevos sentidos las fragmentaciones que como individuo o grupo se han experimentado y por las cuales se han generado traumas, daño y dolor.

Decolonization means being part of a process where you are willing to look at yourself to see the parts that have been affected by White supremacy and check them. [...] We are walking contradictions [...] If we are willing to look at them and to create spaces where this other world is possible, then you are in that process of decolonizing. (Cihuatl Ce, 2010, Intro de canción, Femi- 9mm).

Las raperas entrevistadas buscan construir una contramemoria. Blackwell (2011) lo llama retrofitted memories - o memorias realineadas - y es una manera de reeditar la información de la memoria común al reapropiarse y recrear saberes borrados y fragmentados por las prácticas coloniales de organización del conocimiento y resignificarlos. De esta manera, ellas pueden performar una identidad historizada que produzca fisuras en la 'estabilidad' del sistema de representación dominante, y por lo tanto que abra la posibilidad de desarticularlo. Por esta razón, en sus canciones superponen aspectos que consideran verídicos en distintos periodos históricos - y que también hacen parte de la memoria colectiva - donde se estaba situado en una posición de poder. De este modo, se 'recupera' la capacidad de auto-representación individual y social, lo que posibilita la producción de un cambio de sentido, aunque también de reproducción.

Nuestra gente no vivía con divisiones

No más un pueblo compartiendo la cultura, los valores, las tradiciones

De cada cuatro puntos, cada cuatro direcciones

We were on this land once before united

'till the devil got us all caught up

Now we're divided

15 Al reconocer ese vacío de acción y entender el poder de convocatoria del muchos artistas en la ciudad se le ocurrió organizar Hip Hop for Humanity, un evento que buscaba recolectar comida enlatada para el L.A. Food Bank. Para ello, se propuso reunir material discográfico, camisetas, afiches, y otros productos de artistas locales que quisieran donar. De esta manera, quien traía su donación alimentaria se llevaba en intercambio una bolsa con estos productos. El evento fue un éxito y provocó mucha admiración de los artistas locales que aplaudieron la iniciativa afirmando que se necesita concentrar más trabajo por su parte en la comunidad. 
Norteños, sureños, Crips, Bloods,

Africanos, mexicanos, salvadoreños pandilleros,

Claiming hoods we don 't even own

Fighting over crumbs

While they occupy our home

Now we' re living, now we' re dying

Barely surviving in this poverty

Heartbreaking misery

Look and you will find the answers in your history

It ain 't no mystery

When you now can see

It comes down to this!

So let me see la raza raise the Brown fist to this

Fight for this! Die for this!

Would you risk your life for this? [...]

Black and Brown, Brown and black

Gonna take it all back, click-clack

But first we gotta unite, before we could fight

Eastside, Westside, Southside, no side, all sides, all one side!

'cause it's all about the unity

Starting with our own communities

Understanding who's your enemy $[\ldots]$.

(Cihuatl Ce, 2010, “All Sides”).

En la teoría xicanista, la metodología para descolonizar los saberes y los cuerpos es posicionándose étnica y genéricamente desde lo indígena como el centro mismo del proceso de identificación y reconstitución. Así se abre un espacio para reclamar una experiencia historizada de la articulación de género y etnicidad que resultará en un sujeto político. Esta metodología se encontró en todas las participantes. Algunas de manera explícita en las entrevistas, otras más explícitas en sus canciones. Al performar una raíz femenina imaginada desde la Diosa Madre sagrada y guerrera, este grupo de mujeres busca honrar lo femenino desde perspectivas que recuperan y reconocen saberes que le son propios y que legitiman su estar allí, su derecho a la soberanía sobre su cuerpo, su mente, su espíritu, su comunidad, su ciudad. La noción de honrar debe entenderse como el reconocimiento de la continua e histórica opresión, explotación, y devaluación de lo femenino mediante el ofrecimiento de - para algunas de ellas "ofrendando"sentidos desde donde posicionarse para nombrarse a sí mismas, poder reconciliar y dar resolución a las dualidades coloniales deshumanizantes, sentidos que las empoderan y reivindican.

Esto es pa' las madres fieras, fuertes guerrilleras

vendedoras y fruteras, curanderas, pelioneras

por el bien de sus hijos nunca prisionera ni traicionera

Eso es ella la madre más fieraQueenz de Cuba a Canadá

En todo Anáhuac

hasta las hermanas en Darfur

tous c'est pour la amour

I show sum love for my sistren up in districts

Where they fight for existence

not worried about no lipsticks over instincts

resistance!

Obtener amor sin dolor.

Stop killing our nación de color!

It's your choice to elevate your voice,

Mujer de Zion. Sommes la solution!

(Guerrilla Queenz, (verso de Eyerie), 2011,

"Mucho amor").

De este modo, en una suerte de eficacia simbólica, el sujeto dañado por el sistema opresivo puede curarse al hacer pensable su situación en un acto de representación donde el lenguaje articula signos que relacionan coherentemente la experiencia sensible y orgánica del 'enfermo' con significantes que producen significado, dan sentido a lo que ocurre y esa conciencia inconsciente 'ilumina' el camino hacia una transformación, al desbloqueo, al reordenamiento hacia el bien, hacia la cura (Levi-Strauss,1949, 
pp. 221-223). Todas las raperas entrevistadas construyen el objetivo de su arte en términos de healing, de cura desde lo personal a lo comunal ${ }^{16}$.

Al honrar los valores 'naturales' de lo femenino desde la raíz cultural imaginada, las conexiones con la madre tierra y la valoración del poder creador de la mujer en un nivel sagrado, la eficacia simbólica se traduce en confianza, seguridad y sensación de empoderamiento para otras mujeres. El impacto que este mensaje tiene en las niñas, jóvenes señoras y ancianas de la comunidad sería ya otro problema de investigación a proyectar; lo que sí es claro es que es un objetivo de las artistas.

En el hip hop masculino, este mismo objetivo se cumple pero desde su masculinidad, por lo cual es útil y necesaria la perspectiva de género para profundizar en este fenómeno. Las mujeres que hacen rap saben de estas falencias discursivas y que nadie más va a producir experiencias del barrio desde perspectivas femeninas si las mismas mujeres no lo hacen. Esto les da la fuerza para continuar y seguir luchando por llegar al micrófono y hacerse escuchar. Los contenidos de las canciones, dentro de la dinámica de honrarse a sí mismas y al otro, tratan historias personales, familiares, de la vida cotidiana en el barrio, de la violencia, la pobreza, pero también la esperanza y deseos por una comunidad unida en la lucha por estar siempre mejor.

En términos de la violencia desde una perspectiva femenina, se trata el abuso sexual, el feminicidio, la violencia intrafamiliar. Mientras que los relatos de violencia en el hip hop masculino se sitúan principalmente en las dinámicas de territorialidad en el barrio, estas mujeres cierran esa práctica al nivel del hogar, de las relaciones de pareja, del abuso de poder masculino sobre el femenino cuando la violencia simbólica o psicológica pareciera no ser suficiente.
Most of my music is my perspective as a female. That's why I try to talk about those issues of domestic violence, and the abuse of children, because I want that cycle to end with me and my daughter. That's also why I do the music, to give voice to young girls or women who have experienced that in their own homes! So that we begin to talk about it, 'cause when we can talk about it, we can begin to heal. (Cihuatl Ce, Entrevista directa, 2012).

En el constante proceso de identificación, tanto las ciudades, como los habitantes y los movimientos que conforman están sujetos a la historia y a las transformaciones. El hip hop ha representado un momento histórico del proceso postindustrial de las ciudades globales y se ha convertido en un medio seguro para que los y las jóvenes produzcan presencia y exijan sus derechos por estar allí en la ciudad, ser partícipe de la producción de sentido y provocar los cambios. Pero el hip hop también ha ido reconfigurándose, ha ido corrigiéndose.

We are using hip hop as it has never been used before, because we are identifying ourselves with our indigenous roots, we are talking about indigenismo or reclaiming what is ours, and talking about the social struggles going on! We help to change the face of Hip Hop as well! (Centzi, 33, Entrevista directa, 2012).

En el rap es necesario tener el coraje para hablar, llegar al micrófono y hacer algo valioso de esa experiencia. Se debe trabajar constantemente para desarrollar habilidades, encontrar estilo propio y abrirse el camino. La continua participación de mujeres como estas cinco raperas desplazan las fronteras de la diferenciación sexual; del mismo modo lo hacen otras subjetividades sexuales, étnicas, etcétera. Esto no sólo aporta a la transformación identitaria de género, sino del movimiento cultural en sí. Es un aporte en la consolidación real del hip hop como un foro de discusión, de diálogo.

16 La metodología del feminismo chicano para "curar" las fragmentaciones históricas es la de "bridge" o puente, propuesta por Anzaldúa y Morága en su clásico libro This Bridge Called My Back (1981), pensada como un espacio, un intersticio entre las dicotomías desde donde posicionarse para nombrarse a sí misma y poder reconciliar y dar resolución a las dualidades coloniales deshumanizantes; "[...] cuando pienso en curar y en transformación, pienso en descolonización -la necesidad de crear consciencia sobre las maneras en que las filosofías dominantes occidentales se han impuesto desde el colonialismo" (Ayala et al., 2006, p. 275). Dicho proceso de descolonización puede ser pensado, entonces, como la superación de las formas en que las fragmentaciones del sí mismo han sido internalizadas y naturalizadas, mediante la concientización de otras posibilidades que conformen la propia identidad de una manera integral. 


\section{Conclusiones}

\section{Being xicana means understanding the different parts of the self: yourself, your family, your community.}

(Fe, 2010, WeEP WoMeN)

Las mujeres que han crecido en el barrio postindustrial angelino han estado en constante contacto con el hip hop - por afinidad o no-, pues es uno de los lenguajes dominantes que ha usado la juventud para expresarse en los últimos treinta años. Aquellas que se han interesado en participar activamente en el movimiento, tienen la posibilidad de representar la complejidad del entramado contextual en el que se encuentran desde las experiencias personales y como observadoras y relatoras de la vida cotidiana de su comunidad. En el rap articulan distintas historias, reclamos y críticas que revelan desencuentros cotidianos de triple opresión: por clase, etnia y género; pero también deseos de cambio social.

Además de esto, remiten a contrastes y tensiones que surgen entre generaciones familiares; permiten un acercamiento a las condiciones de vida del barrio; al igual que encarnan una lucha compartida: la reivindicación de lo femenino como un lugar de poder desde donde enunciar su diferencia y renegociar las distintas relaciones sociales que las constituyen en términos de subordinación. La estrategia que han desarrollado es mediante la revisión, reconocimiento y apropiación de saberes que se asocian con una profunda raíz cultural imaginada con los que resignifican lo femenino heredado mediante la revalorización de quiénes son y la transformación de sus prácticas. El nacionalismo cultural de las raperas chicanas dota de otros sentidos la identidad femenina heredada y, por ende, desplaza lógicas de las relaciones de género tradicionales.

En los regímenes androcéntricos el silencio femenino es una cualidad, una virtud, un símbolo de belleza que materializado concreta al cuerpo femenino en un objeto, un enigma a qué la fantasía masculina le da forma; si se interrumpe se rompe esa coherencia y se convierte en algo definido, con perfil propio, algo que incomoda $-\mathrm{y}$ reacomoda- el habitus. Al negar la palabra a las mujeres se les cancela su capacidad de ser en sí mismas, su capacidad creadora y de intervenir creativamente en el mundo.

El rap femenino es desafiante de ese sistema, es tomar el poder de la palabra para producir conocimiento femenino, es enfrentar las fronteras simbólicas y geográficas para desplazarlas y ganar terreno en un mundo de creatividad que les ha sido negado por 'naturaleza'. De esta manera, las dicotomías que fragmentan la experiencia humana en mente/ cuerpo, actividad/pasividad, fortaleza/debilidad, coraje/cobardía, público/privado y que han acompañado históricamente los términos que definen lo masculino y lo femenino, se revelan como un artificio, una arbitrariedad que puede y debe ser redefinida. Ellas luchan por la equidad de género en un campo de valor en donde el talento, el coraje y la creatividad también les pertenezcan, ellas performan la búsqueda de otra mujer posible.

Ya situadas en la palabra, mientras que el interés inicial es captar la atención de su comunidad y mantenerla con el propósito de relatar historias con las que la juventud se pueda identificar, la motivación principal está en la forma de activismo que toma su producción, su chicana hip hop, un hip hop femenino que acompañe a la comunidad hacia un cambio social, una forma de ejercer poder para la transformación de la historia. Esta transformación la construyen en términos de descolonizar $y$ curar. Con el primero buscan desmantelar las nociones ahistóricas y esencialistas con las que se han construido las diferenciaciones de género, étnica, clase y generación que mantienen la opresión por parte del grupo dominante y con el segundo buscan ofrecer otras significaciones para dotar de nuevos sentidos las fragmentaciones que como individuo o grupo se han experimentado y que han causado daño, trauma o dolor. La metodología que usan para la transformación es la de posicionarse étnica y genéricamente desde lo indígena y así reclamar una experiencia historizada desde donde 
honrar lo femenino chicano con saberes que recuperan y reconocen como propios y que las legitima a estar allí en la ciudad como ciudadanas.

Entonces podemos decir que el rap les ofrece un espacio de poder creativo desde donde dirigir sus discursos de cambio y ellas le ofrecen al rap saberes de la vida en el barrio y la ciudad desde lo femenino. Lo que comunican en sus canciones va desde honrar a las mujeres y hombres de su comunidad, de historias personales, familiares, del cuidado de la tierra y sus recursos, de la vida cotidiana de la ciudad, la pobreza, la migración y violencia; pero también hablan de la esperanza por un mejor futuro y de la unidad comunal.

En cuanto a las reflexiones sobre la violencia que viven se puede decir que es uno de los temas donde hay mayor diferenciación entre el rap masculino y el femenino, pues el primero se concentra en una violencia estatal y callejera en una suerte de territorialidad del barrio, mientras que el segundo habla más de la violencia en el hogar, sexual y toca hechos de agresión misógina como el feminicidio. Lo anterior concuerda con el señalamiento de Sassen (2006) cuando dice que hacerse presente o estar presente es reconocer el lugar como eje central de las reclamaciones que articulen el derecho a estar ahí; y en este caso los hombres concentran su reconocimiento el lugar de lo público como su espacio de significación, mientras que las mujeres - a pesar de actualmente disputan una presencia en lo público- concentran su atención de reclamación en el espacio de lo privado, lugar que históricamente les ha 'pertenecido', pero en silencio.

\section{Referencias}

Alarcón, N. (1998). Chicana Feminism: In the Tracks of 'the Native Woman'. En C.Trujillo (ed.), Living Chicana Theory. Berkley: Third Woman Press.

Anzaldúa, G. (1999). Borderlands. La Frontera. The New Mestiza (2a ed.). San Francisco, usa: Aunt Lute Books.
Ayala, J., Herrera, P., Jimenez, L., \& Lara, I. (2006). Fiera, Guambra, Y Karichina!: Transgressing the Borders of Community and Academy. En D. Delgado, A. Elenes, F. Godinez y S. Villenas (eds.), Chicana/Latina Education in Everyday Life: Femenista Perspectives on Pedagogy and Epistemology. Albany, NY: State University of New York Press.

Bauman, Z. (2001). La globalización: sus consecuencias humanas. México: FCE.

Blackwell, M. (2011). ¡Chicana Power! Eeuu: University of Texas Press.

Castells, M. (1989). The Informational City. Blackwell. Oxford-Cambridge (MA).

Castillo, A. (1994). Massacre of the Dreamers: Essays on Xicanisma. Albuquerque: New Mexico University Press.

Cross, B. (1993). It's not about a Salary: Rap, Race and Resistance in Los Angeles. London: Verso.

Davis, M. (2000). Magical Urbanism. Latinos Reinvent the US City. London: Verso.

Davis, M. (1990). City of Quartz. Excavating the future of Los Angeles. Berkeley: University Press Books.

Espín, O. (1999). Language: Identity, Silence and Sexuality. Women Crossing Boundaries. N.Y: Routledge.

Forman, M. (2004). “"Represent”: Race, Space, and Place in Rap Music". En M. Forman y M. McNeal (eds.), That's The Joint. The Hip Hop Studies Reader. N.Y: Routledge

Frith, S. (2003). "Música e identidad”. En S. Hall y P. Gay (eds.), Cuestiones de Identidad. España: Amorrortu.

Johnson, G. T. (2002). "A Sifting of Centuries: Afro-Chicano Interaction and Popular Musical Cultura in California, 1960-2000". En A. Aldama y N. Quiñonez (eds.), Decolonial Voices: Chicano and Chicana Cultural Studies in the 21st Century. Bloomington: Indiana University Press.

Kittay, E. (2010). “The Moral Harm of Migrant Carework: Realizing a Global Right to Care" Polity volume. Gender \& Global Justice, 37(1), 53-73. 
Leite, P., Angoa, A., y Rodríguez, M. (2009). Emigración mexicana a Estados Unidos: balance de las últimas décadas. La situación demográfica de México 2009. México: Consejo Nacional de Población.

Lévi-Strauss, C. (1949). La eficacia simbólica. Antropología estructural. Buenos Aires: Eudeba.

Maier, E. (2001). Las madres de los desaparecidos ¿un nuevo mito materno en América Latina? México: UAM.

Massey, D. S., Alarcon, R., Durand, J., \& Gonzalez, H. (1987). Return to Aztlan: The Social Process of International Migration from Western Mexico. Berkeley: University of California Press.

McFarland, P. (2006). Chicano Rap Roots: BlackBrown Cultural Exchange and the Making of a Genre. Callaloo, 29(3).

McFarland, P. (2008). Chicano Rap: gender and violence in the postindustrial barrio. Texas, EUA: University of Texas Press.

McFarland, P. (2013). The Chican@Hip Hop Nation. Politics of a New Millennial Mestizaje. East Lansing, MI: Michigan State University Press.

Morgan, M. (2009). The Real Hip Hop: Battlinf for Knowledge, Power, and Respect in the L.A. Underground. Durham, NC: Duke University Press Books.

Nateras, A. (2007). "Adscripciones juveniles y violencias transnacionales: cholos y maras". En J. Valenzuela Arce, A. Nateras Domínguez y R. Reguillo Cruz (coords.), Las Maras, Identidades Juveniles al Limite. México: UAM Iztapalapa/Colegio de la Frontera Norte.

Pérez-Torres, R. (2006). Chicano Hip Hop and Postmodern Mestizaje. En The Chicanola Cultural Studies Reader. U.K: Routledge.

Reguillo, R.(2003). "Jóvenesy Estudios Culturales. Notas para un balance reflexivo". En J. M. Valenzuela (ed.), Los Estudios Culturales en México. México: Fondo de Cultura Económica.

Ríos, F. (2008). From Chicano-a to Xicana. Recuperado de http://www.uwyo.edu/edstudies/_files/publications/from_chicanoa_\%20 to_xicanao.pdf
Rose, T. (1994). Black Noise and Black Culture in Contemporary America. Middletown, CT.: Wesleyan University Press.

Sassen, S. (2006). Territory. Authority. Rights. From Medieval to Global assemblages. Princeton University Press.

Sassen, S. (1997). Whose City Is It? Globalization and the Formation of New Claims. Recuperado de http://www.asu.edu/courses/pos445/Sassen-Whose\%20city\%20is\%20it\%3F.pdf

Sassen, S. (2000). Women's Burden: Countergeographies of Globalization and the Feminization of Survival. Journal of International Affairs, Spring 2000, 53 (2).

Sassen, S. (2005). Gobal City: Introducing a Concept. Recuperado de http://www.columbia.edu/ sjs2/PDFs/globalcity.introconcept.2005.pdf

Valenzuela, J. M. (2007). "Cien años de Choledad". En Las Maras, J. Valenzuela Arce, A. Nateras Domínguez y R. Reguillo Cruz (coords.), Identidades Juveniles al Limite. México: UAM Iztapalapa y Colegio de la Frontera Norte.

Velasco, J. (2006). The X in the Race and Gender: Rethinking Chicano/a Cultural Production Through the Paradigms of Xicanisma and $\mathrm{Me}(\mathrm{x})$ icaness. En The Chicano/a Cultural Studies Reader. U.K: Routledge.

\section{Discografía}

Cihuatl Ce. (2010). Not Another Ever Again. En Femi-9mm [Demo]. Los Ángeles, California.

Cihuatl Ce. (2010). All Sides. En Femi-9mm [Demo]. Los Ángeles, California.

Fe. (2010). The Women Weep [Demo]. Los Ángeles, California.

Guerrilla Queenz. (2011). "Mucho Amor" con las Krudas Kubensi [Demo].

Sick Jacken. (2009). Born in L.A. En Stray Bullets [Disco compacto]. Whittier, California: Rebel Music Group.

Tenochtitlan. (2010). 1519. En Obsidian Rapture. Los Ángeles, California: Sin sello discográfico. 